\title{
Flight Test Experiment Design for Characterizing Stability and Control of Hypersonic Vehicles
}

\author{
Eugene A. Morelli* \\ NASA Langley Research Center, Hampton, Virginia, 23681-2199, USA
}

\begin{abstract}
A maneuver design method that is particularly well-suited for determining the stability and control characteristics of hypersonic vehicles is described in detail. Analytical properties of the maneuver design are explained. The importance of these analytical properties for maximizing information content in flight data is discussed, along with practical implementation issues. Results from flight tests of the $X-43 A$ hypersonic research vehicle (also called Hyper-X) are used to demonstrate the excellent modeling results obtained using this maneuver design approach. A detailed design procedure for generating the maneuvers is given to allow application to other flight test programs.
\end{abstract}

\section{Nomenclature}

$\begin{array}{ll}a_{x}, a_{y}, a_{z} & \text { body-axis translational accelerometer measurements, } \mathrm{ft} / \mathrm{sec}^{2} \\ b & \text { wing span, } \mathrm{ft} \\ \bar{c} & \text { mean aerodynamic chord, } \mathrm{ft} \\ I_{x}, I_{y}, I_{z}, I_{x z} & \text { mass moments of inertia, slug- } \mathrm{ft}^{2} \\ k & \text { frequency index } \\ m & \text { number of inputs, or mass, slugs } \\ M & \text { Mach number } \\ m a x & \text { maximum } \\ m i n & \text { minimum } \\ p, q, r & \text { body-axis roll, pitch, and yaw rates, rad/sec } \\ \bar{q} & \text { dynamic pressure, lbf/ft }{ }^{2} \\ r m s & \text { root-mean-square } \\ S & \text { wing reference area, } \mathrm{ft}^{2} \\ T & \text { excitation time length, sec } \\ V & \text { airspeed, ft/sec } \\ x_{c g}, y_{c g}, z_{c g} & \text { coordinates of the center of gravity, in } \\ x_{r e f}, y_{r e f}, z_{r e f} & \text { coordinates of the reference point, in } \\ \alpha & \text { angle of attack, deg } \\ \beta & \text { sideslip angle, deg } \\ \delta_{e}, \delta_{a}, \delta_{r} & \text { symmetric elevon, asymmetric elevon, and rudder deflections, deg } \\ \phi & \text { phase angle, rad } \\ & \\ & \end{array}$

\footnotetext{
${ }^{*}$ Research Engineer, Dynamic Systems and Control Branch, MS 308, Associate Fellow
} 


\section{Introduction}

$\mathrm{O}$ ne of the goals of current aerospace research is to develop vehicles that can maneuver at hypersonic speeds. To achieve that goal, it is necessary to conduct flight tests of hypersonic vehicles to determine their stability and control characteristics. Presently, it is not possible to sustain hypersonic flight at a given flight condition for any substantial length of time, because of limitations in hypersonic propulsion, thermal protection, and practical considerations such as airspace limitations and the size of available test ranges. Consequently, flight conditions are transient when flight testing hypersonic vehicles. In particular, the Mach number changes rapidly and significantly during hypersonic flight tests, which means that any maneuver must be executed efficiently in all axes over a very short time period. This is done so that the flight condition for each maneuver can be considered relatively constant, which simplifies the data analysis and modeling. If the maneuver takes too long, the Mach number changes significantly, and the vehicle aerodynamics, stability, and control change with it.

Stability and control characteristics of a flight vehicle can be quantified by parameters appearing in the equations of motion, such as stability and control derivatives in the case of a linear dynamic model ${ }^{1,2}$. Values of these parameters can be found from flight data using various forms of least squares fitting of the model output (which depends on the values of the model parameters to be estimated) to measured data ${ }^{1}$. For hypersonic vehicles (and many experimental subsonic aircraft as well), flight test maneuvers are executed by applying designed input time series to the control surfaces, using an onboard flight computer ${ }^{3}$. These inputs are perturbations about the nominal or trim control surface deflections.

The requirements for an effective maneuver to determine vehicle stability and control characteristics at hypersonic speeds are as follows:

1) Multiple simultaneous inputs - For maximum flight test efficiency, and to ensure that the vehicle response in all axes is recorded at approximately a constant flight condition, all control surfaces on the vehicle should be moved simultaneously. This approach saves time compared to the conventional approach of moving one control surface at a time in sequence.

2) Orthogonal inputs - With all inputs moving simultaneously for flight test efficiency, it is best if the inputs are mutually orthogonal. Mutual orthogonality means the inputs are the most unlike each other that they could possibly be. Since each input is doing something completely different, the effect of each individual control can be identified as accurately as possible. Considering that the data analysis could be done in either the time domain or the frequency domain, it would be best if this orthogonality could be enforced in both domains.

3) Wide-band frequency content - The best inputs for exciting the vehicle dynamic response would have frequency content at or near the modal frequencies of the vehicle. However, these modal frequencies are only known approximately - if they were known exactly, there would be no need for flight test. A robust input design has wide-band frequency content to cover many possibilities. As long as the frequency content of each input is wide-band, the vehicle dynamic response will be excited, regardless of exactly where the modal frequencies lie. Another practical reason for wide-band inputs is that a single wide-band input design can be applied repeatedly at many different flight conditions, without sacrificing effectiveness. The practical result is a savings in onboard computer memory required, and a simplified input design.

4) Small excursions - The inputs must excite the vehicle dynamic motion, but it is best if this motion can be limited to small excursions from the target flight condition, so that the vehicle stability and control can be adequately described using linear models. For hypersonic flight tests, the requirement for small excursions also arises from the desire to fly the vehicle on a specific planned trajectory. Even small deviations from a planned trajectory can cause large uncertainty in the landing or splashdown location.

5) Perturbation inputs - To achieve small excursions about the target flight condition and keep the vehicle on its intended trajectory, the inputs for the maneuver must be perturbations about the nominal or trim control surface deflections. This means that each input must begin and end at zero amplitude, and oscillate around zero amplitude.

In this paper, a method for designing inputs that meet the above requirements is described in detail. This method, first described in Ref. 3, has been applied successfully to the NASA X-43A (Hyper-X) hypersonic flight tests $^{4,5}$. The form of each input is a sum of sinusoids with unique frequencies and phase shifts. Multiple inputs are 
designed to be mutually orthogonal in both the time domain and the frequency domain, and are optimized for maximum data information content in multiple axes over a short time period, while minimizing excursions from the target flight condition. The only a priori information required for the multiple-input design is an estimate of the approximate frequency band for the vehicle dynamics, and approximate relative control effectiveness for proper relative scaling of the input amplitudes.

The next section describes the input design procedure in detail, along with some analytical results and practical considerations. This is followed by a description of the $\mathrm{X}-43 \mathrm{~A}$ hypersonic research vehicle and flight test, and modeling results from the $\mathrm{X}-43 \mathrm{~A}$ flight data.

\section{Input Design Method}

\section{A. Multiple Input Design}

The general idea is to excite the vehicle using inputs with frequency content near where the modal frequencies of the vehicle dynamic response are expected to lie, using an input form that is efficient for parameter estimation. Each input is a sum of sinusoids with unique frequencies that cover a frequency band of interest, similar to frequency sweeps ${ }^{1}$, and the amplitudes of the sinusoidal components are chosen to achieve a specific power distribution. The wide-band frequency content of the inputs is important because there is naturally some uncertainty as to the exact modal frequencies for the vehicle in flight. Wide-band inputs provide robustness to this uncertainty.

The following material describes in detail how to design multiple orthogonal phase-optimized multi-sines and why this particular input form is efficient for estimating stability and control parameters in dynamic models.

Each perturbation input $\boldsymbol{u}_{j}$, applied to the $j^{\text {th }}$ individual control surface, is comprised of a set of summed harmonic sinusoids with individual phase shifts $\phi_{k}$,

$$
\boldsymbol{u}_{j}=\sum_{k \in\{1,2, \ldots, M\}} A_{k} \sin \left(\frac{2 \pi k \boldsymbol{t}}{T}+\phi_{k}\right) \quad j=1,2, \ldots, m
$$

where $M$ is the total number of available harmonically-related frequencies, $T$ is the time length of the excitation, and $A_{k}$ is the amplitude for the $k^{\text {th }}$ sinusoidal component. The variable $t$ represents a vector of $N$ discrete time points, $\boldsymbol{t}=\left[\begin{array}{lllll}t(0) & t(1) & \ldots & t(N-1)\end{array}\right]^{T}$, and $\boldsymbol{u}_{j}$ represents the vector of corresponding amplitudes for the $j^{\text {th }}$ input, $\boldsymbol{u}_{j}=\left[\begin{array}{llll}u_{j}(0) & u_{j}(1) & \ldots & u_{j}(N-1)\end{array}\right]^{T}$. Each of the $m$ inputs is comprised of selected components from the pool of $M$ sinusoids with frequencies $\omega_{k}=2 \pi k / T, k=1,2, \ldots, M$.

If the phase angles $\phi_{k}$ in Eq. (1) were chosen at random on the interval $[-\pi, \pi] \mathrm{rad}$, then in general, the various harmonic components would add together at some points to produce an input $\boldsymbol{u}_{j}$ with relatively large amplitude excursions. This is undesirable, because it can result in the dynamic system being moved too far from a reference condition selected for the experiment. To prevent this, the phase angles $\phi_{k}$ for each of the selected harmonic components are chosen to minimize relative peak factor $R P F$, defined by ${ }^{1,3,6}$

$$
\operatorname{RPF}\left(\boldsymbol{u}_{j}\right)=\frac{\left[\max \left(\boldsymbol{u}_{j}\right)-\min \left(\boldsymbol{u}_{j}\right)\right] / 2}{\sqrt{2\left(\boldsymbol{u}_{j}^{T} \boldsymbol{u}_{j}\right) / N}}=\frac{\left[\max \left(\boldsymbol{u}_{j}\right)-\min \left(\boldsymbol{u}_{j}\right)\right]}{2 \sqrt{2} \operatorname{rms}\left(\boldsymbol{u}_{j}\right)}
$$

Relative peak factor is a measure of the efficiency of an input for parameter estimation purposes, in terms of the amplitude range of the input divided by a measure of the input energy. The relative peak factor is scaled so that any individual sinusoidal component (such as any one of the summands in Eq. (1)) has $R P F=1$. Low relative peak factors are desirable for estimating dynamic model parameters, because the objective is to excite the dynamic system 
with a variety of frequencies without driving it too far away from the nominal operating point. The target value for optimizing $R P F$ of an input with many harmonic components (cf. Eq. (1)) is 1, to match the $R P F$ for a single harmonic. In some cases, the $R P F$ for a phase-optimized composite input can be slightly less than 1 , as will be shown later. Inputs with low relative peak factors are efficient in the sense of providing good input energy to excite the dynamic system over a selected frequency band, with low amplitudes in the time domain.

For a composite signal with more than one sinusoidal component, as in Eq. (1), the goal is to design the input for minimum $R P F$. This is done by adjusting the phase parameters $\phi_{k}$ for the components of each input to minimize $R P F$. The resulting optimization problem is non-convex; however, a simplex algorithm ${ }^{7}$ can be applied to find a solution.

The integers $k$ specifying the frequencies for the $j^{\text {th }}$ input $\boldsymbol{u}_{j}$ are selected to be unique to that input, but are not necessarily consecutive. A good approach for multiple inputs is to assign integers $k$ to each input alternately. This is illustrated in Figure 1 for the X-43A flight test maneuver design. In this case there were 3 inputs, elevator, aileron, and rudder, and a total of 42 frequencies $(M=42)$. The frequencies were interleaved among the three

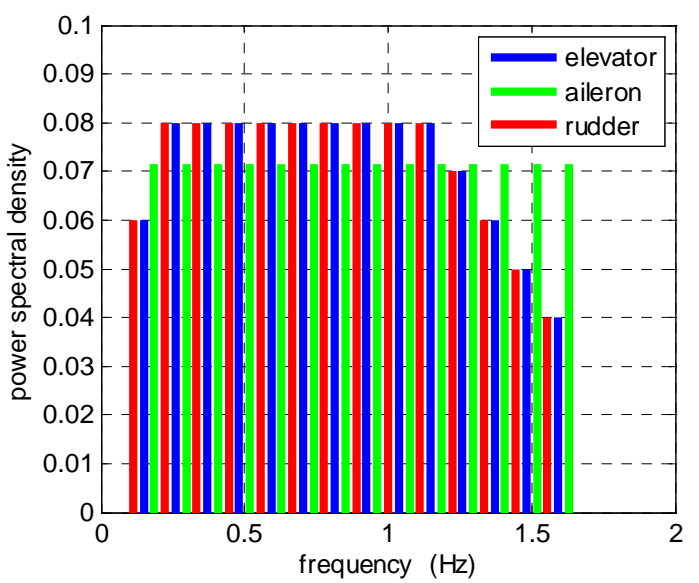

Figure 1 Multiple orthogonal phase-optimized multi-sine input spectra, X-43A inputs to achieve wide-band frequency content for each input. This provided robustness to uncertainty of how each control would excite the dynamic modes of the vehicle. Furthermore, since each input has wideband frequency content, a single input design can be applied at various flight conditions, which simplifies the flight test and requires a small amount of onboard computer memory. The power spectra for elevator and rudder were modified so that more excitation power was applied at middle frequencies where the dynamic modes excited by these controls were believed to be. The power spectra shown are normalized, so the effects of individual control surface amplitudes are excluded. This means that for each input, the sum of all the spectral line ordinates (sum of the heights of the bars for each color) is 1 .

When the frequency indices $k$ that are selected for each input $\boldsymbol{u}_{j}$ in Eq. (1) are distinct from those chosen

for the other inputs, then the frequency content of each $\boldsymbol{u}_{j}$ consists of distinct spectral lines in the frequency

domain, as can be easily seen in Figure 1. Therefore, the vectors of Fourier transforms for the inputs as a function of frequency have inner products equal to zero. In this sense, the inputs are mutually orthogonal in the frequency domain, because each input contains frequencies that no other input has.

In the time domain, a sum of sinusoids is orthogonal to any other sum of sinusoids with harmonically-related frequencies, regardless of the constant phase shift of each sinusoidal component. For example, consider two inputs, each containing a single distinct harmonic sinusoidal component,

$$
\boldsymbol{u}_{1}=\sin \left(\frac{2 \pi k_{1} t}{T}+\phi_{1}\right) \quad \boldsymbol{u}_{2}=\sin \left(\frac{2 \pi k_{2} t}{T}+\phi_{2}\right) \quad k_{1} \neq k_{2}
$$

where $k_{1}$ and $k_{2}$ are both integers. Then using the discrete-time notation $t_{i}=i \Delta t$ and $T=N \Delta t$, where $\Delta t$ is a constant time step, the inner product of these inputs is

$$
\boldsymbol{u}_{1}^{T} \boldsymbol{u}_{2}=\sum_{i=0}^{N-1} \sin \left(\frac{2 \pi k_{1} t_{i}}{T}+\phi_{1}\right) \sin \left(\frac{2 \pi k_{2} t_{i}}{T}+\phi_{2}\right)=\sum_{i=0}^{N-1} \sin \left(\frac{2 \pi k_{1} i}{N}+\phi_{1}\right) \sin \left(\frac{2 \pi k_{2} i}{N}+\phi_{2}\right)
$$


Using the trigonometric identity

$$
\sin (u) \sin (v)=\frac{1}{2}[\cos (u-v)-\cos (u+v)]
$$

Eq. (4) is changed to

$$
\boldsymbol{u}_{1}^{T} \boldsymbol{u}_{2}=\frac{1}{2} \sum_{i=0}^{N-1} \cos \left(\frac{2 \pi\left(k_{1}-k_{2}\right) i}{N}+\phi_{1}-\phi_{2}\right)-\frac{1}{2} \sum_{i=0}^{N-1} \cos \left(\frac{2 \pi\left(k_{1}+k_{2}\right) i}{N}+\phi_{1}+\phi_{2}\right)=0
$$

Each summation in Eq. (6) is zero because the sum of a harmonic sinusoid over the base period $T$ is zero,

$\sum_{i=0}^{N-1} \cos \left(\frac{2 \pi k i}{N}+\phi\right)=0$, for any integer $k$ and any constant phase angle $\phi$. This shows that harmonic sinusoids are orthogonal in the time domain. For more than one sinusoidal component in each input, the analysis is similar. So, inputs assembled as in Eq. (1) are orthogonal in the time domain.

An objective for the experiment design is to excite the aircraft dynamics in a short time period by moving multiple control surfaces simultaneously. Since more than one surface is being moved, it is advantageous for the modeling if the $\boldsymbol{u}_{j}$ vectors applied to each control surface

are mutually orthogonal. This helps the parameter estimation by completely de-correlating the inputs, which improves the accuracy of control effectiveness estimates. Using the design method described here, it is possible to make all of the $\boldsymbol{u}_{j}$ mutually orthogonal in both the time and frequency domains, using inputs designed for minimum relative peak factor. This gives the analyst the flexibility to use either time domain or frequency domain parameter estimation methods while retaining the desirable feature of mutually orthogonal inputs.

Figure 2 shows perturbation inputs applied at Mach 6 on the X-43A. These inputs are mutually orthogonal in both the time and frequency domains. The inputs were computed from Eq. (1), using the spectra shown in Figure 1, with phase angles $\phi_{k}$ optimized for minimum relative peak factor. Table 1 contains all the information for the input design at Mach 6. Because of the various frequencies and phase angles, and the small amplitudes of the input perturbations, applying these inputs simultaneously to the vehicle produces a dynamic response similar to what might be seen in flight through light to moderate turbulence. Consequently, the vehicle stays on
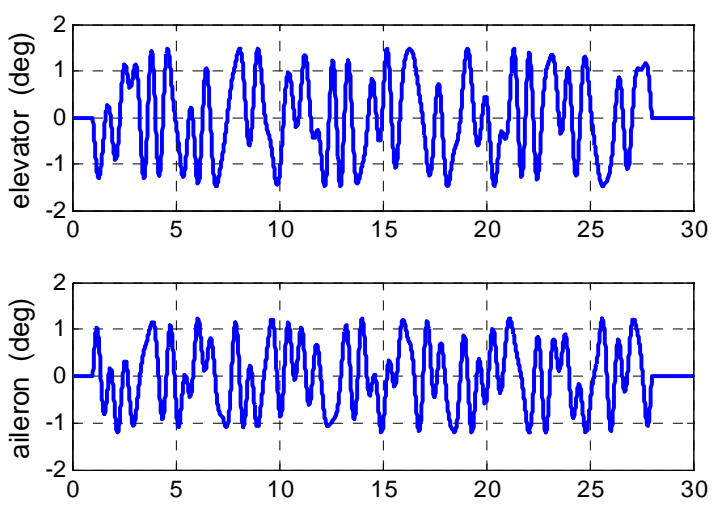
its nominal trajectory, but jiggles around that.

For each input, the power spectrum can be tailored by selecting the $A_{k}$ to distribute power over the spectral components. To achieve a uniform power distribution,

$$
A_{k}=\frac{A}{\sqrt{n}} \quad \forall k
$$


where $n$ is the number of sinusoidal components included in the summation of Eq. (1), and $A$ is the amplitude of the composite input $\boldsymbol{u}_{j}$. Therefore, with uniform power distribution, selection of the $A_{k}$ reduces to selecting a single input amplitude $A$.

To summarize, the multiple input design procedure is as follows:

1) 1. Select the time period $T$ for the excitation, which determines the smallest harmonic frequency resolution $\Delta f=1 / T \mathrm{~Hz}$ and the limit on the minimum frequency, $f_{\min } \geq 2 / T \mathrm{~Hz}$. Often this is dictated by practical considerations such as length of time available at a particular flight condition.

2) Select the frequency band of the dynamic system for the excitation frequencies, $\left[f_{\min }, f_{\max }\right] \mathrm{Hz}$. This corresponds to the frequency band where the dynamic response of the system is expected to lie. The frequencies are equally spaced by $\Delta f$ on the interval $\left[f_{\min }, f_{\max }\right]$. The total number of frequencies is $M=f i x\left[\left(f_{\max }-f_{\min }\right) / \Delta f\right]+1$, where $f i x$ indicates rounding to the nearest integer toward zero.

3) Assign approximately an equal number of indices $k$ from the set $\{1,2, \ldots, M\}$ to each input by alternating each consecutive frequency among the multiple inputs. This approach produces lower relative peak factors for the individual inputs, and also ensures that each input has frequency content distributed evenly across the frequency band $\left[f_{\text {min }}, f_{\text {max }}\right]$. Different assignments of the frequency indices could be made for other reasons. For example, lower frequency indices might be assigned to an input that is known to effectively excite a low frequency mode, or particular frequency indices might be omitted to avoid exciting an undesirable structural response or to simplify the input design. Each frequency index can be assigned to only one of the inputs, to preserve mutual orthogonality of the inputs in both the time and frequency domains.

4) Generate the input $\boldsymbol{u}_{j}$ for each of the $m$ controls, using Eq. (1) and assigning the starting values for the phase angles $\phi_{k}$ at random from a uniform distribution on the interval $[-\pi, \pi] \mathrm{rad}$.

5) Choose the input power spectrum by specifying the amplitude $A_{k}$ for each harmonic component, or use Eq. (7) to implement a uniform power distribution.

6) Apply a simplex optimization algorithm ${ }^{8}$ to adjust the $\phi_{k}$ for each $\boldsymbol{u}_{j}$ to achieve minimum relative peak factor for that input. The simplex optimization technique does not require gradients.

7) For each input, do a one-dimensional search to find a constant time offset for the components of each input $\boldsymbol{u}_{j}$, so that the input begins and ends at zero amplitude. This is equivalent to sliding the input along the time axis until a zero crossing is placed at the origin of the time axis. The appropriate phase shift is added to each component phase angle $\phi_{k}$. Note that to implement a constant time shift to all the components, the phase offset for each component will be different, because each component has a different frequency. Since the components of each $\boldsymbol{u}_{j}$ are harmonics of the base frequency with period $T$, if all the component phase angles $\phi_{k}$ are shifted so that the initial value of the input is zero, then the final value of the input at time $T$ will also be zero. The power spectrum, input orthogonality, and relative peak factor are all unaffected.

8) Return to step 6 unless the relative peak factor has reached a pre-defined goal value or a maximum number of iterations has been reached. For practical work, the relative peak factor goal is set at 1.01 and the maximum number of iterations is set to 50 . 


\section{B. Practical Issues}

All of the steps detailed above are implemented in MATLAB ${ }^{\circledR}$ code that is part of a software package called SIDPAC (System IDentification Programs for AirCraft). SIDPAC is bundled with Ref. 1. SIDPAC also contains many other useful programs for experiment design, data analysis, modeling, and simulation. The program that implements the above steps is called mkmsswp.m.

Sometimes repeating the input design with different random starting values for the $\phi_{k}$ (cf. step 4 above) can lead to an improved (lower) optimized relative peak factor solution. This behavior is common for high-dimensional non-convex optimization problems such as this one. In practice, this just means re-running the input design program, because each run uses a different realization of the random initial values for $\phi_{k}$.

To gain an appreciation of the non-convexity of the optimization problem, Figure 3 shows the relative peak factor as a function of phase angles for two components in the summation of Eq. (1), using $T=15$ sec, unit amplitudes, and frequency indices $k=2$ and $k=4$. It is clear from the figure that the optimization problem is not convex, and also that there are several phase angle solutions which are equally good, or nearly so. The data used to make Figure 3 can be used

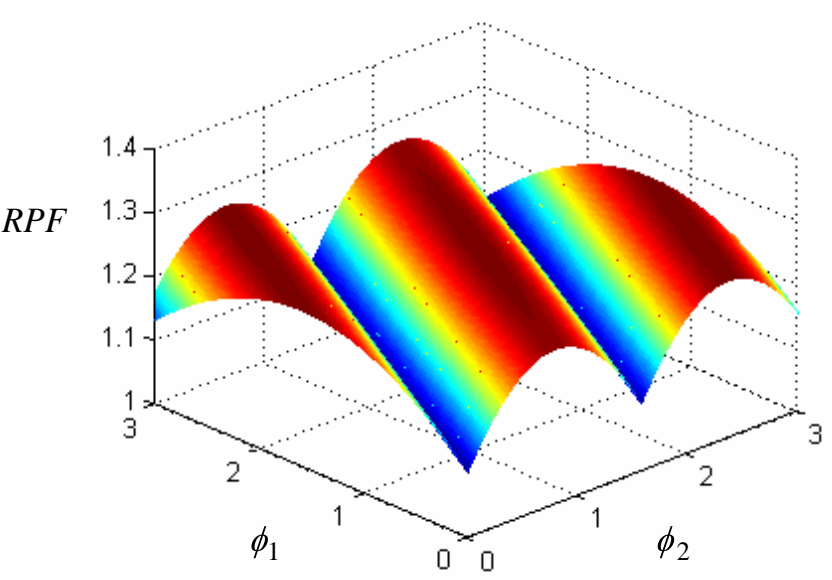

Figure 3 Relative peak factors for a two-component multi-sine input for a global exhaustive search for the minimum relative peak factor in this simple case. To generate this data, the relative peak factors were computed for values of the phase angles on a 2-dimensional grid with intervals of $0.0175 \mathrm{rad}(1 \mathrm{deg})$, over the range $[0, \pi] \mathrm{rad}([0,180] \mathrm{deg})$. The minimum relative peak factor of all these computed values was found to be 1.102. Using the technique described above to optimize the phase angles, the relative peak factor achieved was 1.106. This demonstrates that the technique developed here found an input with relative peak factor very close to the global optimum, for this simple case. The same exercise was then repeated for three harmonic components, with frequency indices $k=2,4$, and 6 , and the same phase angle intervals, in three dimensions. In this case, the global exhaustive search gave a minimum relative peak factor equal to 1.002 , and the optimization technique described above produced an input with relative peak factor equal to 1.002. These investigations suggest that the optimization procedure does an excellent job of designing inputs with relative peak factor very close to the global optimum.

Step 7 of the input design procedure ensures that each designed input will be a perturbation, so that the designed input can be added to whatever value the control may have for another reason, e.g., trim or maneuvering. The iteration in this step helps to reduce the relative peak factors by adding perturbations to the phase angles $\phi_{k}$ (from the phase changes added to make the input start and end at zero), which helps the optimization algorithm solve the non-convex optimization problem.

It was found empirically that if the set of selected harmonic frequency indices $k$ selected for a particular input consisted of an integer greater than 1 , along with 2 or 3 multiples of that integer (e.g., $k=2,4,6$, or $k=5,10,15,20$ ), the phase angles could be optimized (in step 6) so that the relative peak factor for that input was very close to 1 , and sometimes less than 1 .

Time length of any input can be extended or reduced, without affecting the orthogonality properties or the relative peak factors, by simply re-computing each input from Eq. (1) using the same optimized phase angles $\phi_{k}$ and amplitudes $A_{k}$, but a different value of $T$. This is equivalent to stretching or shrinking each harmonic component. Of course, if $T$ is changed, the frequency content of the input changes. As $T$ increases, the frequencies in the input shift lower, with decreased frequency spacing. This is demonstrated in Figure 4 for a single 
optimized input form with frequency indices $k=2,4,6,8$. The phase angles of the harmonic components for each of the inputs shown in Figure 4 are the same, and the relative peak factor of each input is 0.995 .
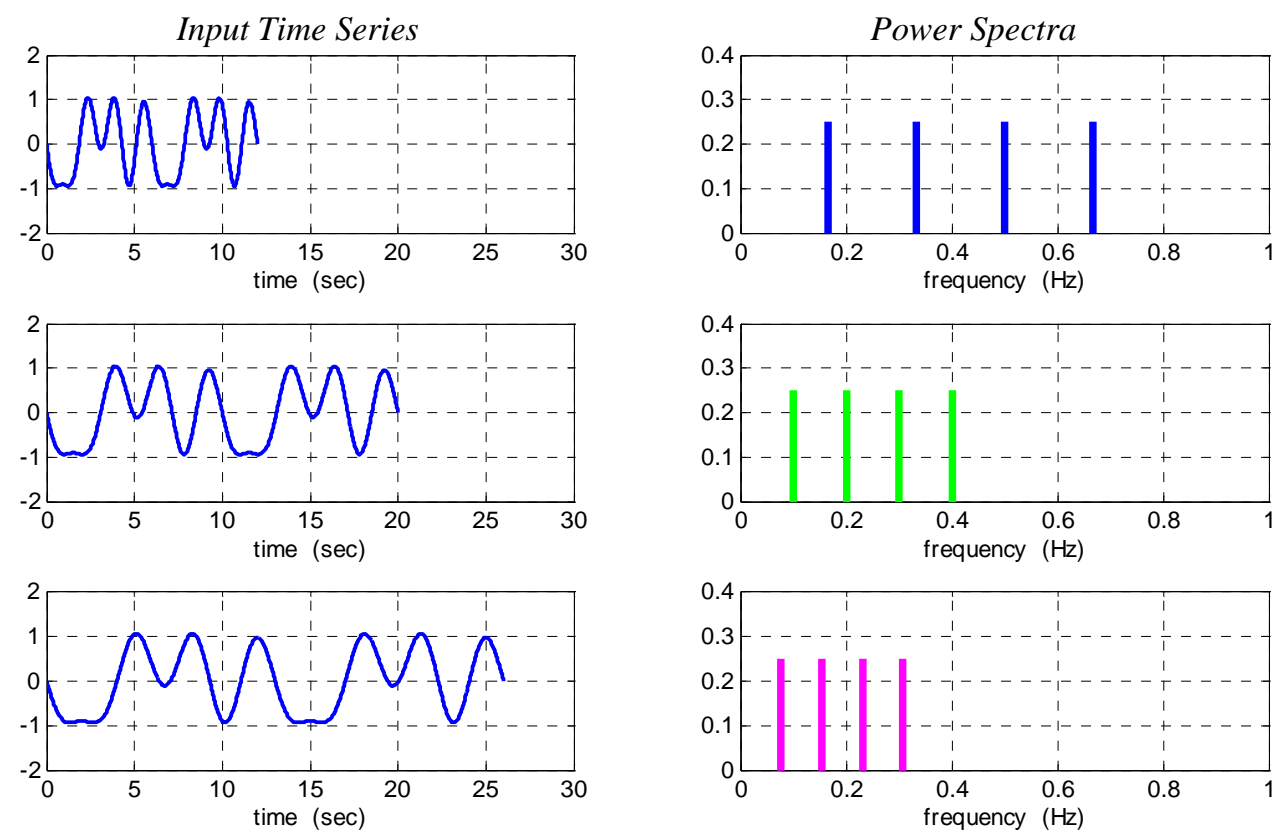

Figure 4 Effect of changing excitation time $T$

It is possible to choose non-integer values of $k$ when assembling the inputs using Eq. (1). This allows arbitrary spacing of the frequencies for each input. As long as unique frequencies are assigned to each input $\boldsymbol{u}_{j}$, mutual orthogonality in the frequency domain remains intact. However, Eqs. (3)-(6) and the associated discussion show that inputs with non-integer values of $k$ will not be mutually orthogonal in the time domain unless the sum and difference of the frequency indices are all equal to an integer. It may still be advantageous to use non-integer values of $k$ in some cases, because even though the inputs will not be orthogonal in the time domain, their correlation will still be low, and this situation is still quite acceptable for good modeling results. Furthermore, if the vehicle has a feedback control system operating during the experiment, then the time-domain orthogonality of the inputs is ruined anyway, by the actions of the feedback control. Assuming the resulting input correlation is not too high (absolute value of the correlation must be below 0.9 ), the modeling results will not be adversely affected.

Although there are methods for optimizing the frequency spectrum of inputs for parameter estimation (i.e., the choice of $A_{k}$ in Eq. (1)), all of them require some knowledge of the vehicle dynamics, usually in terms of a nominal dynamic model with parameter values. For hypersonic flight tests, there is questionable value to such methods, because there is typically significant uncertainty associated with pre-flight predictions of hypersonic vehicle dynamics. Under these circumstances, it is best to define the frequency spectra for all inputs to be relatively flat across the selected frequency band, so that the aircraft dynamics will be sufficiently excited, regardless of where the modal frequencies happen to be located within the frequency band.

The next two sections describe how the input design method explained above was applied to flight testing the X-43A (Hyper-X) hypersonic vehicle. 


\section{Research Vehicle and Experimental Data}

On November 16, 2004, flight 3 of the NASA X-43A hypersonic research vehicle, or Hyper-X, was successfully conducted over the Pacific Ocean off the California coast. The flight included a successful test of the scramjet

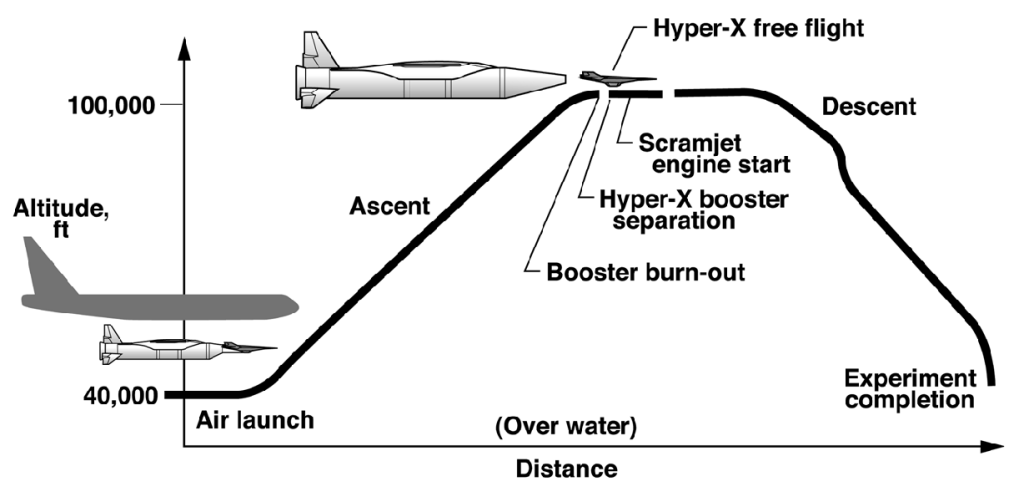

Figure 5 Hyper-X mission profile

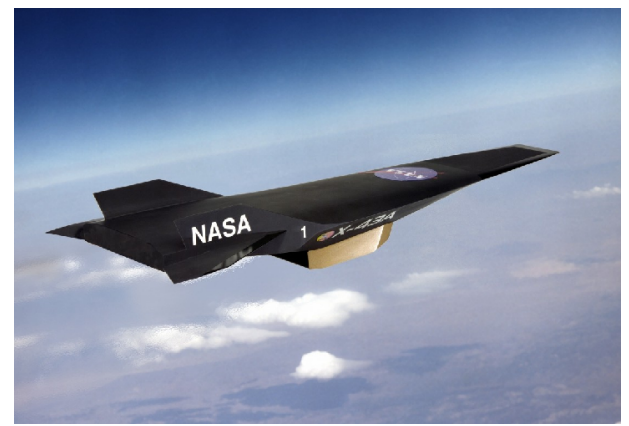

Figure 6 X-43A research vehicle propulsion system at approximately Mach 10. Following the engine test, the engine cowl door was closed, and a series of maneuvers were executed for aerodynamic parameter estimation as the vehicle descended and Mach number decreased. Maneuvers were executed for flight conditions at Mach 8, 7, 6, 5, 4, and 3 . The vehicle flew as a glider (engine off) throughout these maneuvers. Figure 5 shows the $\mathrm{X}-43 \mathrm{~A}$ mission profile. Figure 6 is an artist's rendering of the X-43A research vehicle in flight. A 3-view drawing is shown in Figure 7.

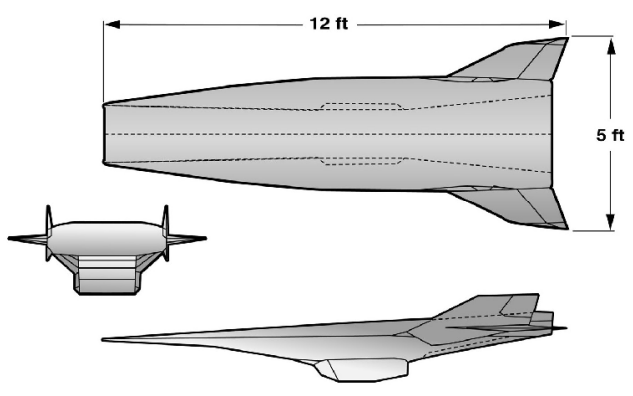

Figure $7 \mathrm{X}-43 \mathrm{~A}$ research vehicle 3-view

At the start of the mission, the X-43A research vehicle was mounted to the nose of a modified Pegasus booster. This configuration, called the Hyper-X stack, was carried aloft by the NASA B-52B aircraft in a manner similar to the X-1 flight tests. Figure 8 is a picture of the Hyper-X stack being carried to altitude for flight 3 . At 40,000 ft, the Hyper-X stack was dropped, and the Pegasus booster fired about 5 seconds later. The Pegasus booster carried the $\mathrm{X}-43 \mathrm{~A}$ to the target flight condition for the scramjet engine test, which was approximately Mach 10 and 100,000 ft altitude. At that point, the X-43A separated from the Pegasus booster, and began its free flight. After stabilization at the target flight condition, the engine cowl was opened and the scramjet engine test was conducted. Then, the engine cowl door closed, and the $\mathrm{X}-43 \mathrm{~A}$ executed an energy reduction maneuver to arrive at the Mach 8 flight condition. Perturbation inputs for aerodynamic parameter estimation, control law validation, and aerodynamic prediction were then run automatically, and these were repeated at Mach 7, 6, 5, 4, 3, and 2 during the vehicle descent. The form of these inputs (shown in Figure 2) was the same at each Mach number, but the amplitude of the inputs changed with flight condition. The vehicle then splashed into the Pacific ocean and was not recovered. Note that the data at Mach 2 was not usable for parameter estimation, because at that flight condition, the vehicle encountered wind shears and gusts which were unmeasured and therefore corrupted the parameter

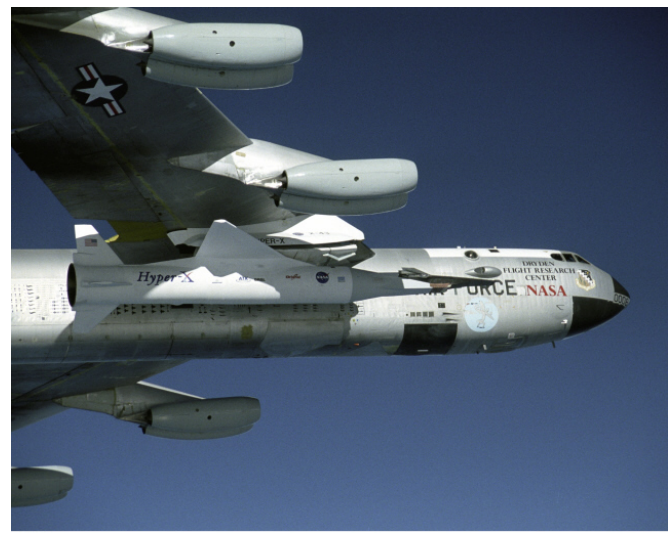

Figure 8 Hyper-X stack being carried to altitude by the NASA B-52B 
estimation results. Consequently, flight 3 results did not include the Mach 2 flight condition.

Ref. 8 includes complete information on the geometry and mass properties of the X-43A research vehicle. Table 2 gives a summary of the constant mass and geometry values used for analysis of the descent data. The mass properties reflect the fully loaded X-43A research vehicle minus the mass of fuel and coolant used during the scramjet engine test.

Flight data came from the Best Estimated Trajectory (BET), documented in Refs. 8 and 9. The BET data set used the best data from several recorded sources, corrected for known time skews and estimated winds aloft. The BET data were also corrected for systematic instrumentation errors using a data compatibility analysis. Sample rate for the BET flight data was $50 \mathrm{~Hz}$, corresponding to a sampling interval of $0.02 \mathrm{sec}$.

All parameter estimation was done for the aerodynamic forces and moments acting at the model reference point, which is the reference point for the aerodynamic database. This was done to facilitate comparisons between stability and control derivatives from the aerodynamic database and estimates based on flight data. The same approach was used for the flight 2 results documented in Ref. 4. Details of the location of the model reference point are available in Ref. 8. Coordinates of the reference point are given in Table 2, for a standard right-handed coordinate frame using fuselage station, butt line, and water line coordinates, as defined in Ref. 8. The aerodynamic reference point is about 5.5 feet aft of the nose, on the vehicle centerline.

\section{Flight Test Results}

The descent maneuvers were designed using the procedure described in Section II. Multiple orthogonal phase-optimized multi-sine inputs were applied to all three control surface inputs - symmetric elevon (elevator), asymmetric elevon (aileron), and rudder. Since flight conditions change rapidly during the descent, the inputs were designed to be applied simultaneously, so that a full set of non-dimensional longitudinal and lateral stability and control derivatives could be estimated at each flight condition. The flight testing also included multi-step 2-1-1 waveforms applied to each of the control surface inputs in sequence, following the multi-sine inputs. Flight data from the 2-1-1 maneuvers were used to test the prediction capability of the models identified from the multi-sine inputs. Accurate prediction for maneuvers with dissimilar inputs is a strong indicator of good modeling results.

Figure 2 shows the multiple orthogonal phase-optimized multi-sine perturbation inputs that were applied at Mach 6. The same input forms were used at every flight condition from Mach 8 to Mach 3, but the amplitudes of the inputs were adjusted to account for the changing dynamic pressure and control surface effectiveness. This approach was taken because of a practical limitation on flight computer memory. The wide-band frequency content of the inputs made it possible to satisfy this practical constraint without compromising the effectiveness of the excitation.

As noted earlier, orthogonal inputs are advantageous for parameter estimation experiments, because the aerodynamic dependencies can be assigned more accurately when all inputs are doing something different to excite the dynamic response of the vehicle. The orthogonality of the designed inputs was disrupted during the flight testing of the X-43A, due to the action of the feedback control, which could not be turned off. However, starting out with orthogonal inputs prevented the feedback control from correlating the inputs to the point where the modeling results would be adversely impacted. In effect, since the inputs were orthogonal (i.e., perfectly decorrelated) to start with, the degradation by the feedback control only reduced them to very good (i.e., very low correlation).

Amplitudes for the three inputs were selected based on test runs of a nonlinear simulation of the Hyper-X, which used an aerodynamic databas $\mathrm{e}^{10}$ built from extensive wind tunnel tests and CFD, and included the feedback control system. The aim was to scale the inputs to achieve response amplitudes of approximately \pm 3 deg in angle of attack and sideslip angle perturbations for each maneuver, based on the simulation. Input amplitude adjustments were done by trial-and-error, because the feedback control system distorted the perturbation inputs for parameter estimation in a manner that depended on the vehicle response. Note that the form of the perturbation input commands did not change - this form was optimized by the procedure described earlier. The trial-and-error was only for sizing the amplitudes of the three inputs at the various flight conditions from Mach 8 to Mach 3.

Standard routines from the software toolbox called System IDentification Programs for AirCraft (SIDPAC $)^{1}$ were used to identify the appropriate model structure for the aerodynamic forces and moments, and to estimate the parameters in the identified model structures. Details on how this was done can be found in Refs. 1, 3, and 4. 
For all flight conditions from Mach 8 to Mach 3 during the descent, a linear aerodynamic model was found to be adequate to characterize the measured data. The aerodynamic model equations were

$$
\begin{gathered}
C_{L}=C_{L_{\alpha}} \alpha+C_{L_{\delta_{e}}} \delta_{e}+C_{L_{o}} \\
C_{D}=C_{D_{\alpha}} \alpha+C_{D_{\delta_{e}}} \delta_{e}+C_{D_{o}} \\
C_{m}=C_{m_{\alpha}} \alpha+C_{m_{q}} \frac{q \bar{c}}{2 V}+C_{m_{\delta_{e}}} \delta_{e}+C_{m_{o}} \\
C_{Y}=C_{Y_{\beta}} \beta+C_{Y_{\delta_{r}}} \delta_{r}+C_{Y_{o}} \\
C_{l}=C_{l_{\beta}} \beta+C_{l_{\delta_{a}}} \delta_{a}+C_{l_{\delta_{r}}} \delta_{r}+C_{l_{o}} \\
C_{n}=C_{n_{\beta}} \beta+C_{n_{\delta_{a}}} \delta_{a}+C_{n_{\delta_{r}}} \delta_{r}+C_{n_{o}}
\end{gathered}
$$

where all state and control variables are perturbations from a reference condition, defined at the beginning of each maneuver.

Lift and drag coefficients were used instead of the body-axis $X$ and $Z$ components of aerodynamic force, so that the flight results could be compared directly to values from the pre-flight aerodynamic database ${ }^{10}$. Aerodynamic moment coefficients were modeled at the aerodynamic reference point used for the aerodynamic database, again to facilitate comparisons.

For all flight conditions studied, the pitch rate damping was the only rate derivative term that had a significant effect on the X-43A aerodynamics. Model terms associated with other rate derivatives were dropped during model structure determination, because the models given in the above equations almost completely characterized the variations in the associated force or moment coefficient. This simply means that rate damping terms were not necessary to model the lift, drag, side force, or lateral moment coefficients, so the damping terms were omitted from those models. Equivalently, the damping parameters were zero for these force and moment coefficients, based on the flight data.

The aerodynamic model parameters in Eqs. (8)-(13) can be estimated from flight data using linear regression or output-error maximum likelihood in the time-domain or frequency-domain, among other approaches ${ }^{1}$. Software that implements each of these methods is included in SIDPAC, and these tools were applied to the flight data. The most consistent results, which also exhibited the best prediction capability, came from equation-error in the frequency domain. Consequently, all of the parameter estimation results shown in this work were computed using equation-error in the frequency domain. Detailed information on this approach can be found in Refs. 1, 3, and 4.

The equation-error method calculates aerodynamic parameter estimates that minimize squared errors between values of the non-dimensional force and moment coefficients determined from measured flight data, and model values computed from Eqs. (8)-(13). For a glider (e.g., the X-43A in descent), non-dimensional force and moment coefficients are determined from measured flight data using the following equations:

$$
\begin{gathered}
C_{X} \equiv-C_{A}=\frac{m a_{x}}{\bar{q} S} \\
C_{Y}=\frac{m a_{y}}{\bar{q} S}
\end{gathered}
$$




$$
\begin{gathered}
C_{Z}=-C_{N}=\frac{m a_{z}}{\bar{q} S} \\
C_{L}=-C_{Z} \cos \alpha+C_{X} \sin \alpha \\
C_{D}=-C_{X} \cos \alpha-C_{Z} \sin \alpha \\
C_{l}=\frac{I_{x}}{\bar{q} S b}\left[\dot{p}-\frac{I_{x z}}{I_{x}}(p q+\dot{r})+\frac{\left(I_{z}-I_{y}\right)}{I_{x}} q r\right] \\
C_{m}=\frac{I_{y}}{\bar{q} S \bar{c}}\left[\dot{q}+\frac{\left(I_{x}-I_{z}\right)}{I_{y}} p r+\frac{I_{x z}}{I_{y}}\left(p^{2}-r^{2}\right)\right] \\
C_{n}=\frac{I_{z}}{\bar{q} S b}\left[\dot{r}-\frac{I_{x z}}{I_{z}}(\dot{p}-q r)+\frac{\left(I_{y}-I_{x}\right)}{I_{z}} p q\right]
\end{gathered}
$$

Substituting measured data into the right sides of Eqs. (14)-(21) results in $N$ values of the non-dimensional force and moment coefficients, where $N$ is the number of data points for the maneuver. Using these values and measured states and controls in Eqs. (8)-(13) results in an over-determined set of equations for the unknown aerodynamic parameters, which can be solved with standard least-squares methods ${ }^{1,3,4,7}$.

Estimates for stability and control derivatives associated with the lift coefficient are plotted in Figure 9 as a function of Mach number. The triangles represent flight estimates, and the circles are values computed from table look-ups in the pre-flight aerodynamic database. Error bars on all estimates indicate \pm 2 standard errors, corresponding to $95 \%$ confidence, assuming a Gaussian distribution. Error bars for the aerodynamic database values represent an estimated uncertainty, computed based on the uncertainty model documented in Ref. 11. Error bars for the flight estimates were statistical values based on the flight data.

Numbers on vertical axes for the parameter estimate plots have been removed, because the numerical results are restricted to U.S. government employees and U.S. government contractors only. However, the scales have not been altered, so the relative positions of the estimates and sizes of the error bars are real.

Similar plots for pitching moment coefficient parameters and drag coefficient parameters are shown in Figures 10 and 11, using the same presentation format. Side force, yawing moment, and rolling moment coefficient parameters appear in Figures 12, 13, and 14, respectively. Inspection of these plots shows that the flight estimates were generally in good agreement with values obtained from the pre-flight aerodynamic database. This is indicated by flight estimates usually lying within the uncertainty bounds of the corresponding value from the aerodynamic database. The flight estimates had small error bars, indicating accurate parameter estimates, which is the direct result of effective experiment design, including both instrumentation and the input design for the maneuvers. The standard errors for the flight estimates were less than 5 percent of the estimated values, for all parameters except the pitch rate derivative $C_{m_{q}}$. No adjustments were made to the flight estimates or the flight data analysis procedure to improve the match to the aerodynamic database. Flight estimates and their error bounds were computed first, and separately, then simply plotted with the values from the pre-flight aerodynamic database as Figures 9-14.

The pitch damping $C_{m_{q}}$ in Figure 10 shows what looks like a significant mismatch between flight estimates and the aerodynamic database. However, the $C_{m_{q}}$ parameter multiplies the quantity $q \bar{c} / 2 \mathrm{~V}$ in the model equations, cf. Eq. (10). At Mach 5, for example, the value of $q \bar{c} / 2 \mathrm{~V}$ is 0.0006 for $q=0.5 \mathrm{rad} / \mathrm{sec}$, which means that the value of $C_{m_{q}}$ must be quite large in order for the damping term $C_{m_{q}} \frac{q \bar{c}}{2 V}$ to be a non-trivial contributor in the model of 
Eq. (10). In fact, the estimates of $C_{m_{q}}$ shown in Figure 10 were small in magnitude, so that the mismatch seen for the $C_{m_{q}}$ estimates makes very little difference in the pitching moment computed from Eq. (10). The possibility of a numerical problem in the parameter estimation arising from the relatively small value of $q \bar{c} / 2 \mathrm{~V}$ compared to the other explanatory variables in the model was examined and ruled out.

Modeling results from flights 2 and 3 could not be compared for maneuvers executed at similar Mach numbers, because the flight conditions were different. Consequently, it was more meaningful to compare flight 3 estimates to values extracted from the aerodynamic database at the same flight conditions, rather than to compare flight estimates for flights 2 and 3. The maneuvers at similar Mach numbers on flights 2 and 3 had dissimilar values of important flight condition parameters such as nominal angle of attack and elevator deflection. Agreement of flight 2 parameter estimation results with values from the pre-flight aerodynamic database (cf. Ref. 4) was very similar to that shown in Figures 9-14 for flight 3.

Figure 15 shows a typical model fit to the flight data. This particular example is the model fit to the pitching moment coefficient at Mach 5 on flight 3. The excellent fit quality is typical of the other cases as well. The upper plot in Figure 15 shows the model fit to the data in the frequency domain, the middle plot shows the model fit to the data in the time domain, and the lower plot shows the difference between the two traces in the middle plot, which is the residual in the time domain. The residual shows no deterministic content, indicating that the model has captured all of the deterministic variation. Note that the time-domain model includes a time-domain least-squares estimate of bias and linear trend with time in the measured output, because these terms are omitted when modeling in the frequency domain.

In Figures 16 and 17, the model identified using flight data from a maneuver designed as described above was used to predict the pitching moment coefficient at a similar Mach number (approximately Mach 5), using a different input, namely a 2-1-1 multi-step. Figure 16 shows the Mach number variation over the time corresponding to the parameter estimation input (done first), followed by a control system validation sweep at higher frequencies, then finally the 2-1-1 prediction maneuver. The upper plot in Figure 17 shows an expanded plot of the 2-1-1 command to the symmetric elevon appearing at the end of the sequence in Figure 16, along with the measured symmetric elevon position. The commanded symmetric elevon was modified by feedback control. The middle plot in Figure 17 shows the time-domain prediction of pitching moment coefficient and the lower plot is the residual, or the difference between the two traces in the middle plot. The quality of the model fit to the prediction data is similar to that seen in Figure 15, indicating that the identified model is a good predictor for a dissimilar maneuver at similar flight conditions. Good prediction capability is a very strong indicator of an accurate model. The quality of the prediction result shown in Figure 17 was typical of the prediction results for other flight conditions.

Slight mismatches toward the end of the prediction maneuver in Figure 17 might be attributable to changes in the vehicle aerodynamics due to rapidly changing Mach number during the descent. Figure 16 shows that the Mach number variation over the period of time from the parameter estimation input to the 2-1-1 prediction maneuver is approximately -0.75 . The model identified from data near the start of the time period may be slightly inapplicable for the prediction maneuver executed near the end of the time period.

\section{Concluding Remarks}

A multiple-input design technique for characterizing the stability and control of hypersonic vehicles was described and demonstrated. The technique can be used to design multiple inputs that are mutually orthogonal in both the time and frequency domains. Mutual orthogonality of the inputs is important for accurate modeling results, and also improves flight test efficiency and practicality, because orthogonal inputs can be applied simultaneously. This allows all relevant data to be collected at an approximately constant flight condition, simplifying the data analysis and modeling. Since the maneuvers are designed for selected frequency ranges, there is no need for a priori models in the design process, and the same input forms can be used over a wide range of flight conditions. In addition, the inputs are optimized for minimum amplitude excursions in the time domain for a given input energy. Power spectra for the inputs can be chosen to target or exclude specific frequencies or frequency bands. These features make the inputs practical and useful for hypersonic flight tests, where the excitation must have short duration and low output response amplitudes, and modal frequencies vary with rapidly changing flight conditions. 
The practical application of the multiple input design technique was demonstrated by estimating stability and control parameters for the X-43A hypersonic research vehicle from flight data. Maneuvers were flown using multiple orthogonal phase-optimized multi-sine inputs applied as simultaneous control surface perturbations at Mach 8, 7, 6, 5, 4, and 3 during the vehicle descent on flight 3. All model parameters, consisting of non-dimensional longitudinal and lateral stability and control derivatives, were estimated based on flight data from a single multi-axis maneuver executed at each Mach number. Multi-step inputs at nearly the same flight conditions were also flown to assess the prediction capability of the identified models. Parameter estimates were plotted as a function of Mach number, and compared with estimates from a pre-flight aerodynamic database, which was built from extensive wind-tunnel tests and computational fluid dynamics. Accuracy of the flight-determined modeling results was significantly better than the estimated uncertainty in the pre-flight aerodynamic database.

Multiple orthogonal phase-optimized multi-sine inputs were shown to be good practical inputs for determining stability and control characteristics of hypersonic vehicles. These wide-band inputs provided sufficient excitation to the X-43A in spite of a feedback control system that distorted the desired input forms. The resulting flight data had sufficient information content for the extraction of highly accurate estimates of aerodynamic stability and control derivatives. Standard errors for the aerodynamic parameters estimated from flight data were less than 5 percent for all parameters except the pitch damping derivative. Flight data from multi-step 2-1-1 maneuvers at similar flight conditions were used to show that the models identified using data from the multiple orthogonal phase-optimized multi-sines had good prediction capability for maneuvers with dissimilar input forms. All of these results support high confidence in the models identified from flight data.

In addition to assessing vehicle stability and control, aerodynamic parameters estimated from hypersonic flight data are useful for comparisons with results from wind tunnel tests and computational fluid dynamics (CFD). This helps to validate and refine aerodynamic prediction methods for designing future hypersonic vehicles. Flight-determined aerodynamic parameters can also be used to update aerodynamic databases for hypersonic vehicles, for improved simulation, control system design, and dynamic analysis. Of course, the multiple orthogonal phase-optimized multi-sine inputs can be applied very effectively to other dynamic systems as well, for many of the same reasons outlined here for hypersonic vehicles.

Because of the success of the X-43A flight tests and the practical considerations discussed earlier, the input design method described in this paper is being applied to the upcoming hypersonic flight testing for stability and control characterization on the U.S. Air Force / DARPA FALCON project ${ }^{12}$.

\section{References}

${ }^{1}$ Klein, V. and Morelli, E.A. Aircraft System Identification - Theory and Practice, AIAA Education Series, AIAA, Reston, VA, August 2006.

${ }^{2}$ McRuer, D., Ashkenas, I. and Graham, D. Aircraft Dynamics and Automatic Control, Princeton University Press, Princeton, NJ, 1973.

${ }^{3}$ Morelli, E.A. "Multiple Input Design for Real-Time Parameter Estimation in the Frequency Domain," Paper REG-360, $13^{\text {th }}$ IFAC Symposium on System Identification, Rotterdam, The Netherlands, August 2003.

${ }^{4}$ Morelli, E.A., Derry, S.D., and Smith, M.S. "Aerodynamic Parameter Estimation for Flight 2 of the X-43A," Paper No. 20, Joint Army Navy NASA Air Force (JANNAF) Conference, Charleston, SC, June 2005 (classified).

${ }^{5}$ Morelli, E.A., Derry, S.D., and Smith, M.S. "Aerodynamic Parameter Estimation of the X-43A (Hyper-X) from Flight Test Data," AIAA-2005-5921, AIAA Atmospheric Flight Mechanics Conference, San Francisco, CA, August 2005.

${ }^{6}$ Barker, H.A., Rivera, D.E., Tan, A.H., and Godfrey, K.R. "Perturbation Signal Design," Paper FrA5.1, $14^{\text {th }}$ IFAC Symposium on System Identification, Newcastle, Australia, March 2006, pp. 1121-1126.

${ }^{7}$ Press, W.H., S.A. Teukolsky, W.T. Vettering, and B.R. Flannery Numerical Recipes in FORTRAN: The Art of Scientific Computing, $2^{\text {nd }}$ Ed., Cambridge University Press, New York, NY, 1992, Chapter 10.

${ }^{8}$ Del Corso, J.A. "X-43A Flight 3 Data Release,” HX-1077, May 2005.

${ }^{9}$ Karlgaard, C. and Tartabini, P.V. "Best Estimated Trajectory Reconstruction for the X-43A Mach 7 and 10 Flights," Paper No. 17, Joint Army Navy NASA Air Force (JANNAF) Conference, Charleston, SC, June 2005. 
${ }^{10}$ Engelund, W. C., Holland, S. D., Cockrell, C., E., Bittner, R. D. “Aerodynamic Database Development for the Hyper-X Airframe Integrated Scramjet Propulsion Experiment," Journal of Spacecraft and Rockets, Vol. 38, No. 6, pp. 803-810, Nov-Dec, 2001.

${ }^{11}$ White, J.T. "HXRV First-Flight Aerodynamic Uncertainty Model," DFRC-HX-0082, November 1999.

${ }^{12}$ Walker, S.H. and Rodgers, F. "Falcon Hypersonic Technology Overview," AIAA-2005-3253, AIAA/CIRA 13th International Space Planes and Hypersonics Systems and Technologies, Capua, Italy, May 2005. 
Table 1 Multiple input design for the X-43A flight 3 descent, Mach 6, $T=27 \mathrm{sec}$

\begin{tabular}{|c|c|c|c|c|c|}
\hline Input & $A(\mathrm{deg})$ & $A_{k}(\operatorname{deg})$ & $k$ & $\phi_{k}(\mathrm{rad})$ & $R P F$ \\
\hline \multirow{14}{*}{$\delta_{e}$} & \multirow{14}{*}{1.0} & 0.3062 & 4 & 0.1356 & \multirow{14}{*}{1.179} \\
\hline & & 0.3536 & 7 & 2.7368 & \\
\hline & & 0.3536 & 10 & 3.5216 & \\
\hline & & 0.3536 & 13 & 3.4517 & \\
\hline & & 0.3536 & 16 & 1.2786 & \\
\hline & & 0.3536 & 19 & 3.1393 & \\
\hline & & 0.3536 & 22 & 4.3436 & \\
\hline & & 0.3536 & 25 & 4.6801 & \\
\hline & & 0.3536 & 28 & 2.7439 & \\
\hline & & 0.3536 & 31 & -0.1261 & \\
\hline & & 0.3307 & 34 & 2.6789 & \\
\hline & & 0.3062 & 37 & 2.4822 & \\
\hline & & 0.2795 & 40 & 0.8446 & \\
\hline & & 0.2500 & 43 & 4.3635 & \\
\hline \multirow{14}{*}{$\delta_{a}$} & \multirow{14}{*}{0.4} & 0.2673 & 5 & 3.1024 & \multirow{14}{*}{1.215} \\
\hline & & 0.2673 & 8 & 3.1671 & \\
\hline & & 0.2673 & 11 & 0.7765 & \\
\hline & & 0.2673 & 14 & 4.1085 & \\
\hline & & 0.2673 & 17 & -1.0068 & \\
\hline & & 0.2673 & 20 & 0.9306 & \\
\hline & & 0.2673 & 23 & -0.0231 & \\
\hline & & 0.2673 & 26 & 4.4717 & \\
\hline & & 0.2673 & 29 & 0.4577 & \\
\hline & & 0.2673 & 32 & 0.8148 & \\
\hline & & 0.2673 & 35 & 1.8706 & \\
\hline & & 0.2673 & 38 & 0.2096 & \\
\hline & & 0.2673 & 41 & 4.5988 & \\
\hline & & 0.2673 & 44 & -0.1935 & \\
\hline \multirow{14}{*}{$\delta_{r}$} & \multirow{14}{*}{5.0} & 1.4697 & 3 & 3.8424 & \multirow{14}{*}{1.029} \\
\hline & & 1.6971 & 6 & 4.0608 & \\
\hline & & 1.6971 & 9 & 3.4827 & \\
\hline & & 1.6971 & 12 & 0.7669 & \\
\hline & & 1.6971 & 15 & 3.2681 & \\
\hline & & 1.6971 & 18 & 2.8489 & \\
\hline & & 1.6971 & 21 & 2.0128 & \\
\hline & & 1.6971 & 24 & 0.6371 & \\
\hline & & 1.6971 & 27 & 2.7851 & \\
\hline & & 1.6971 & 30 & -1.4702 & \\
\hline & & 1.5875 & 33 & 3.6098 & \\
\hline & & 1.4697 & 36 & 2.5226 & \\
\hline & & 1.3416 & 39 & -1.3918 & \\
\hline & & 1.2000 & 42 & 1.2756 & \\
\hline
\end{tabular}


Table 2 Geometry and mass properties of the X-43A research vehicle for flight 3 descent

\begin{tabular}{|c|c|}
\hline \hline $\bar{c}, \mathrm{ft}$ & 12.00 \\
\hline$b, \mathrm{ft}$ & 5.19 \\
\hline$S, \mathrm{ft}^{2}$ & 36.144 \\
\hline$x_{r e f}$, in & 66.240 \\
\hline$y_{r e f}$, in & 0.000 \\
\hline$z_{r e f}$, in & 0.000 \\
\hline$x_{c g}$, in & 69.193 \\
\hline$y_{c g}$, in & 0.019 \\
\hline$z_{c g}$, in & -0.547 \\
\hline mass $m$, slugs & 87.75 \\
\hline$I_{x}$, slugs- $\mathrm{ft}^{2}$ & 54.46 \\
\hline$I_{y}$, slugs- $\mathrm{ft}^{2}$ & 851.40 \\
\hline$I_{z}$, slugs- $\mathrm{ft}^{2}$ & 880.37 \\
\hline$I_{x z}$, slugs- $\mathrm{ft}^{2}$ & 23.51 \\
\hline \hline
\end{tabular}



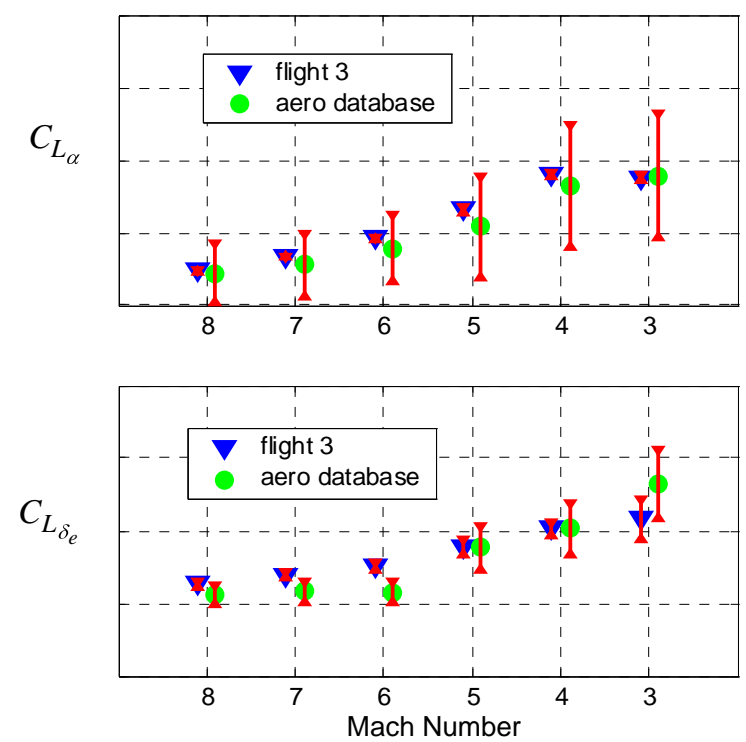

Figure 9 Lift parameters
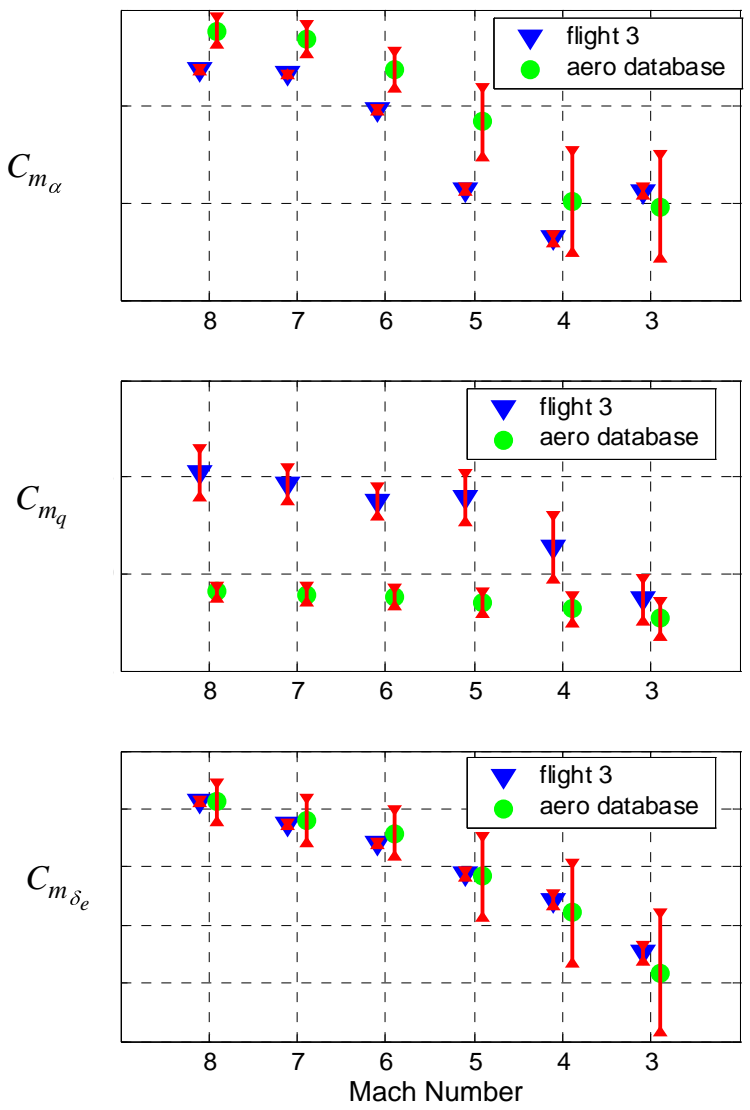

Figure 10 Pitching moment parameters
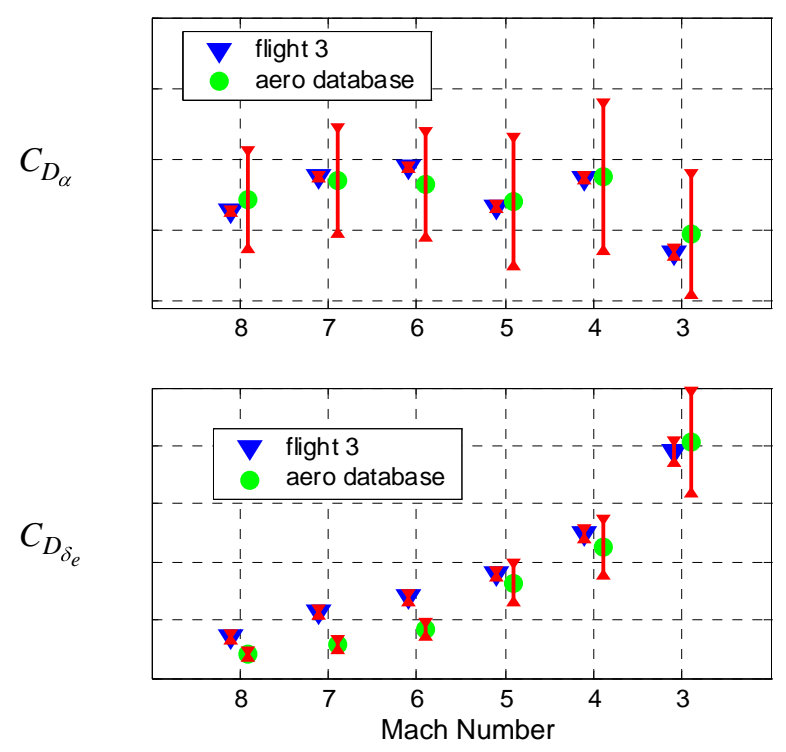

Figure 11 Drag parameters 

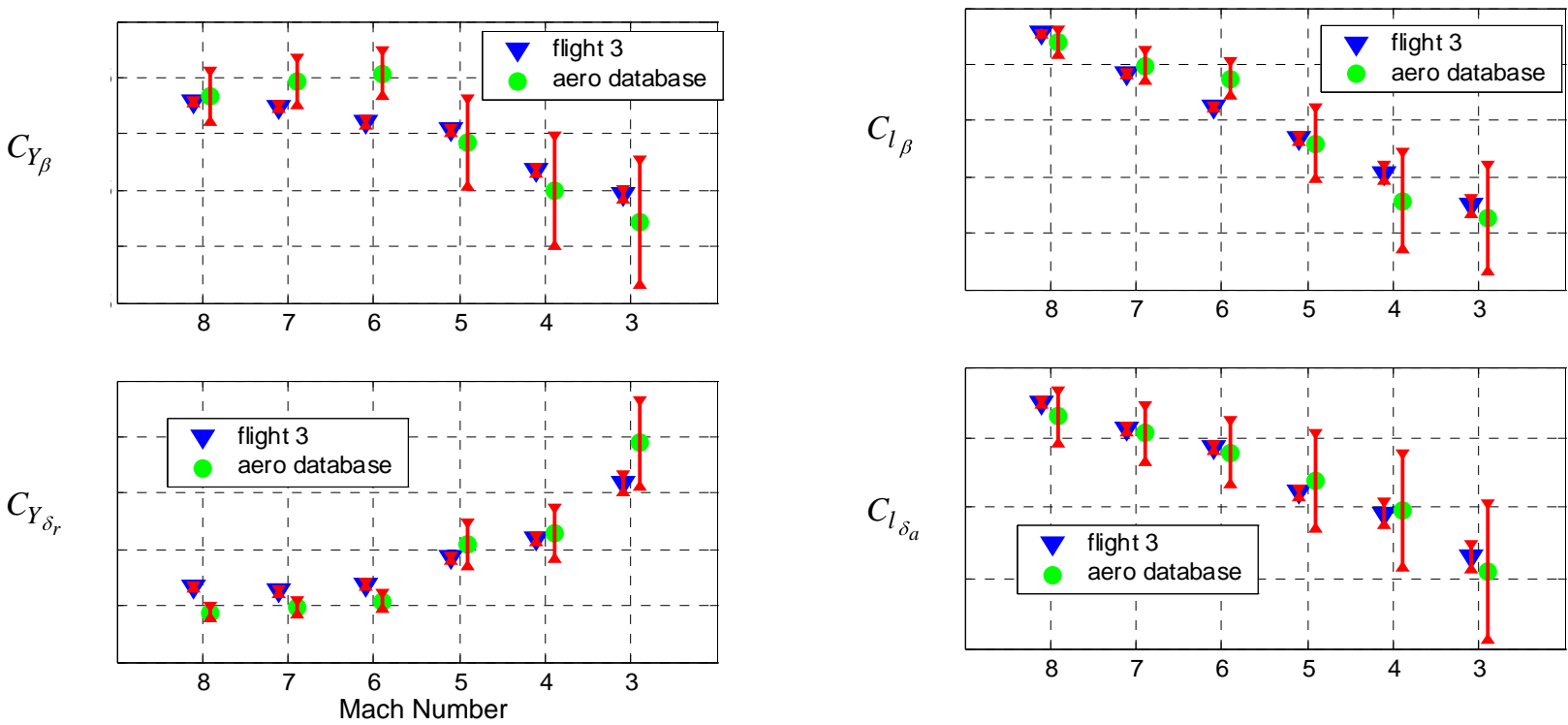

Figure 12 Side force parameters
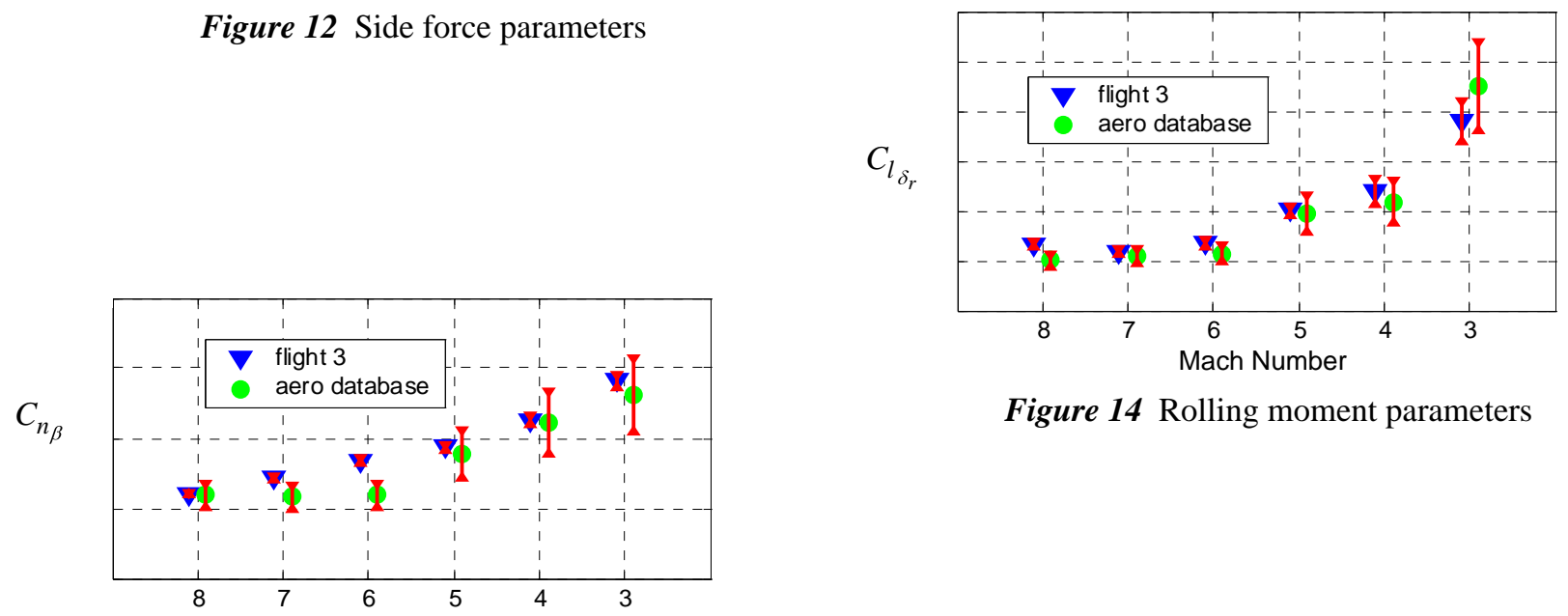

Figure 14 Rolling moment parameters
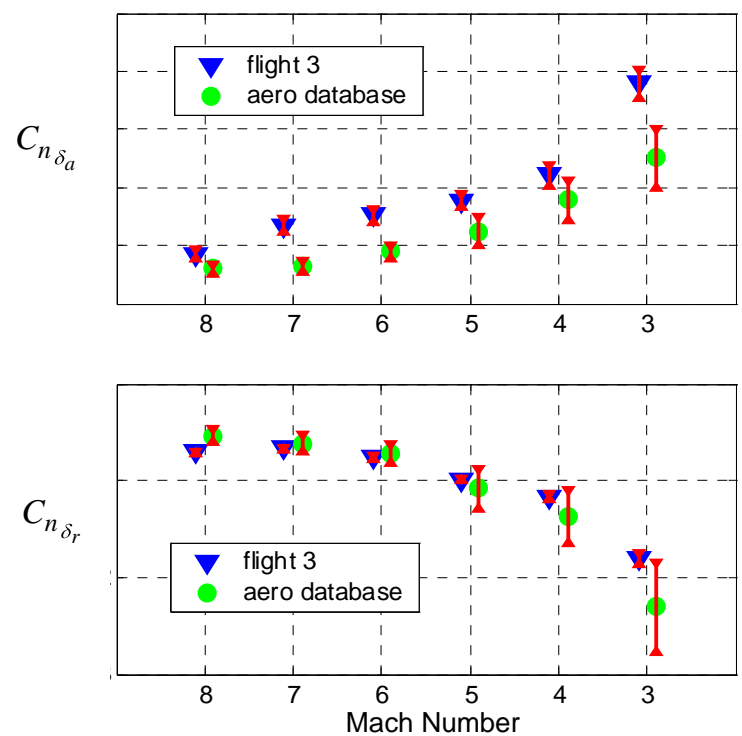

Figure 13 Yawing moment parameters 


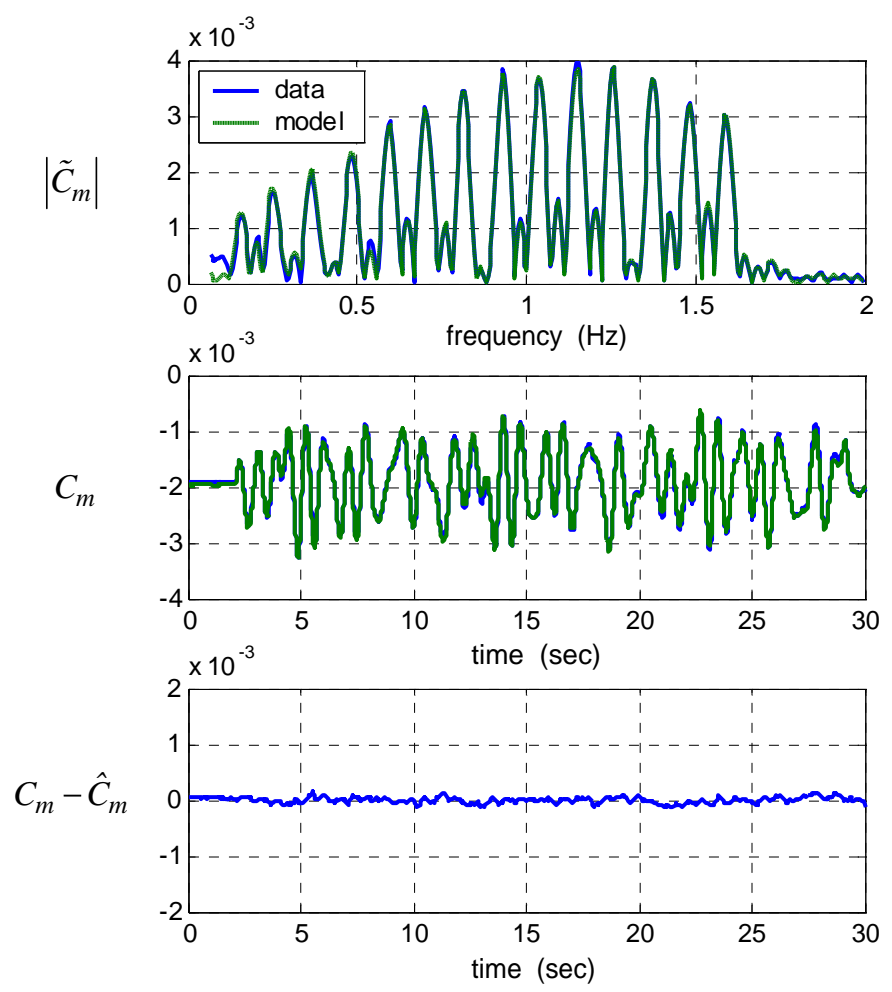

Figure 15 Model fit to pitching moment data, Mach 5
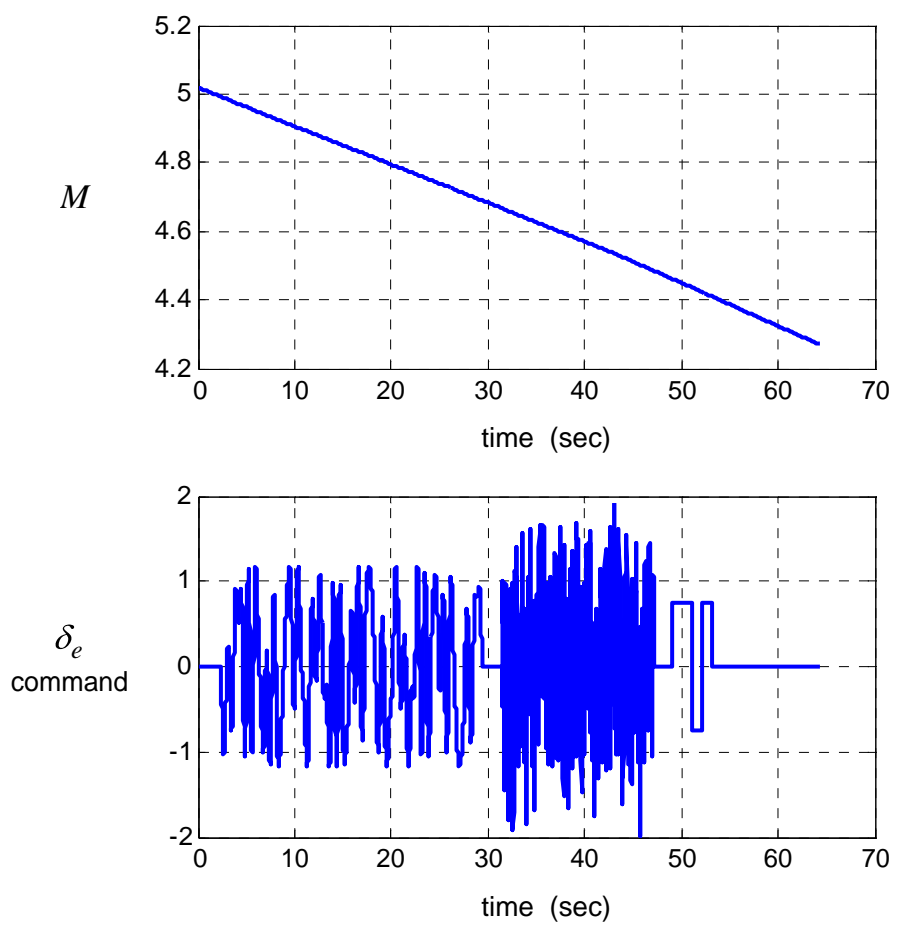
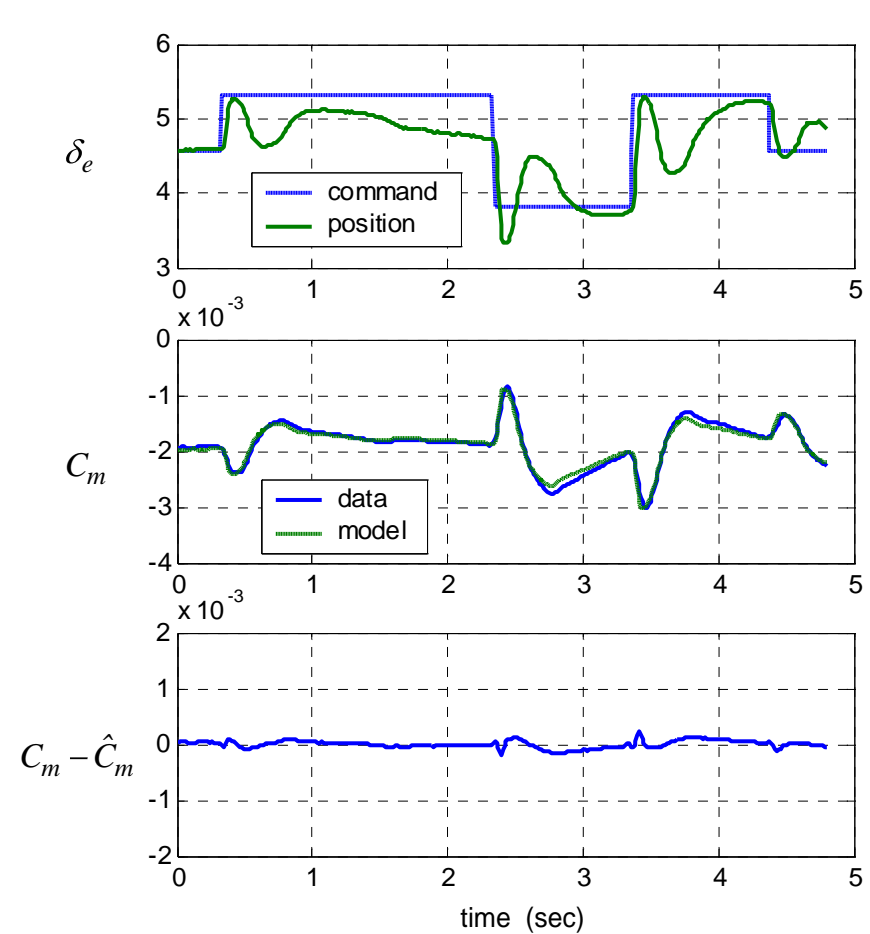

Figure 17 Model prediction of pitching moment data, Mach 5

Figure 16 Mach number variation, Mach 5 maneuvers 